\title{
Variation in practice: should we be standardising diabetes care to improve quality?
}

\author{
PAUL S GRANT
}

\begin{abstract}
It is recognised that there are highly variable outcome and complication rates for patients with diabetes across the UK. The practice of healthcare professionals has been transformed by a growing evidence base quantifying the benefits and risks of interventions in diabetes and the development of clinical practice guidelines summarising this evidence base. Interventions which target the system of chronic disease management, along with patient mediated quality improvement strategies, are important factors significantly contributing to improvements in diabetes care and outcomes. This article explores this phenomenon and demonstrates some of the key quality improvement work that has been done in this area.
\end{abstract}

Key words: diabetes, quality improvement

\section{Introduction}

The Atlas of Variation in Healthcare for People with Diabetes in the UK is an interesting, if not controversial piece of work in that it provides us with a stark overview of how highly variable outcomes and complications rates can be for patients with diabetes across the country (Figure 1). ${ }^{1}$ There are of course many reasons behind this variance, be they demographic, socio-economic, organisational or political, but what is clear is that when it comes to developing services to reduce such variations and attempts to improve quality, collectively we are not winning.

Over the last two decades, the practice of healthcare professionals has been transformed by a growing evidence base quantifying the benefits and risks of interventions in diabetes and the development of clinical practice guidelines summarising this evidence base. One could argue that this has eroded the traditional autonomy of prescribers to choose the treatment that they considered right for 'their' patient, but considerable variation in the

Consultant Physician Diabetes \& Endocrinology, Diabetes \& Endocrine Day Centre, St. Thomas' Hospital, Westminster Bridge Road, London, UK

Address for correspondence: Dr Paul S Grant

Diabetes \& Endocrine Day Centre, St. Thomas' Hospital, Westminster Bridge Road, London, SE1 9EH, UK

Tel: $+44(0) 2071887188$

E-mail: drpaul.grant@doctors.org.uk

Br J Diabetes Vasc Dis 2014;14:30-34

http://dx.doi.org/10.15277/bjdvd.2014.006

\author{
Abbreviations and acronyms \\ DKA \\ GMC \\ $\mathrm{HbA}_{1 \mathrm{c}}$ \\ HES \\ NICE \\ QI \\ QOF \\ diabetic ketoacidosis \\ General Medical Council \\ glycated haemoglobin \\ Hospital Episode Statistics \\ National Institute for Health and Clinical Excellence \\ quality improvement \\ quality outcomes framework
}

Figure 1. The Atlas of Variation in Healthcare for People with Diabetes

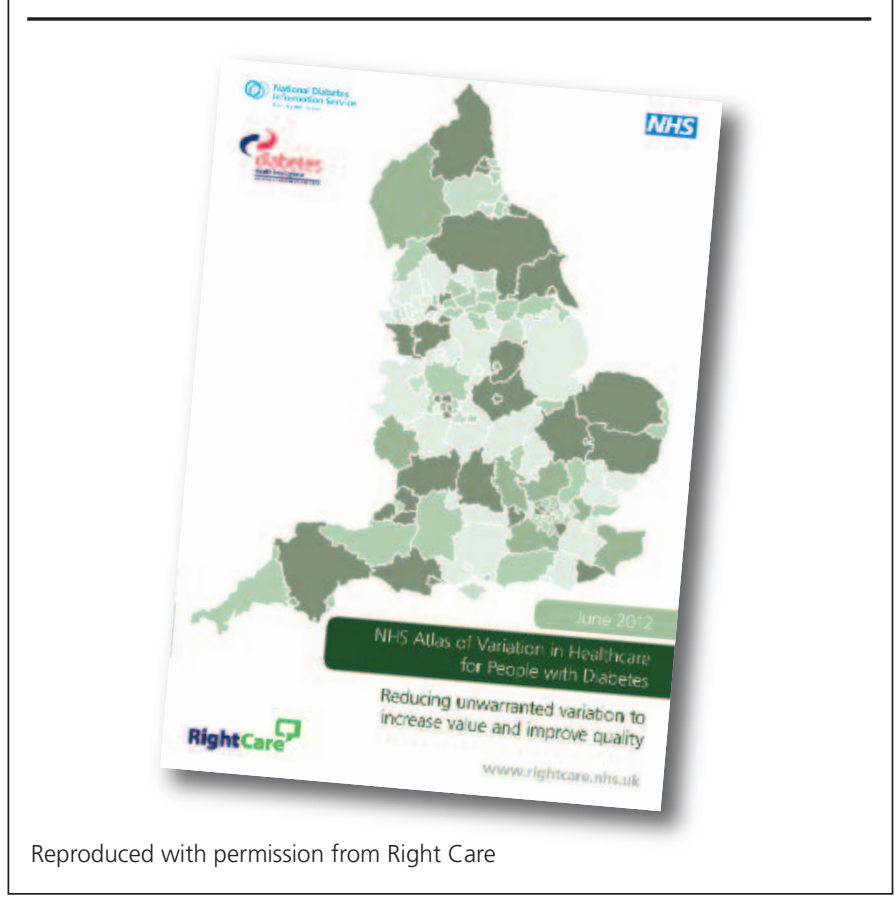

quality of care delivered to patients across the NHS seems to persist. 2,3 The suggestion is that many patients experience clinical encounters having not been offered treatments for their condition for which there is high-quality evidence (grade, level 1).

A systematic review and meta-analysis of QI strategies on the management of diabetes ${ }^{4}$ noted that interventions which targeted the system of chronic disease management, along with patient mediated QI strategies, were important factors significantly contributing to improvements in diabetes care and outcomes (Box 1).

Interventions which solely target healthcare professionals only seem to be beneficial if the baseline $\mathrm{HbA}_{1 \mathrm{c}}$ was poor and 


\section{Box 1 Proven contributing factors in diabetes QI}

- Case management

- Team changes

- Electronic patient registries

- The facilitated relay of information to clinicians

- Continuous QI

- Audit and feedback

- Clinical education

- Clinician reminders

- Financial incentives

- Patient education and self-management

- Reminder systems

in this context one could argue that any intervention will be advantageous. ${ }^{4,5}$

\section{Variation in care}

The NICE quality standards for the management of diabetes were released in 2011; these were 14 statements that described what high quality care for patients should look like (Box 2). ${ }^{6}$

\section{Box 2 NICE diabetes quality standards}

\section{Statement}

1. At diagnosis - structured education programme for diabetes patients and/or their carers, with annual review and access to ongoing education

2. Diabetes patient to receive personalised nutrition and physical activity advice from suitably trained professional or within their education programme.

3. Diabetes patient involved in annual care planning resulting in agreed and documented goals and action plan.

4. Documented agreement on personalised $\mathrm{HbA}_{1 \mathrm{c}}$ target (usually 48 $\mathrm{mmol} / \mathrm{mol}-58 \mathrm{mmol} / \mathrm{mol} ; 6.5 \%-7.5 \%$ ), and patient to receive ongoing review of treatment to minimise hypoglycaemia.

5. Diabetes patient to agree with their healthcare professional to adhere to NICE guidance regarding use of antidiabetic, antihypertensive and lipid modifying medication.

6. Initiation and management of insulin therapy within a structured programme that includes dose titration by the person with diabetes.

7. Women of childbearing age with diabetes be regularly informed of the benefits of pre-conception glycaemic control, offered preconception care or contraceptive advice - including risks to self and unborn child.

8. Annual assessment for risk and presence of diabetes complications; with these to be managed appropriately.

9. Diabetes patients to be assessed for psychological problems and managed appropriately.

10. Diabetes patients at risk of foot ulceration to receive regular review by a foot protection team providing NICE guided treatment.

11. Diabetes patients with foot problems needing urgent attention be referred to and treated by a multidisciplinary foot care team within 24 hours.

12. In-hospital patients with diabetes be provided access to a specialist diabetes team, and given the option of self-monitoring and managing their own insulin.

13. Following DKA, patient to receive educational and psychological support prior to discharge and be followed-up by a specialist diabetes team.

14. Following hypoglycaemia requiring medical attention, patient to be referred to a specialist diabetes team.
However there was a lack of detail within these standards and there was no formal back-up system of logging, recording or auditing whether these standards have been met by a diabetes service. Certainly there was no clear discussion about how they would be implemented into practice or monitored by an internal or external body. They therefore met with a degree of criticism from observers ${ }^{7}$ and are not routinely addressed by practitioners or used to develop a service specification by commissioners.

The inherent goal was to help with standardisation of care delivery and there is often a tension between standardisation and the need to allow innovation and local excellence. Often the failure to offer treatments for which there is the highest quality (level 1) evidence is caused by systems that are not designed for reliable delivery of care and are highly susceptible to human factors. $^{8}$

Service re-design is recognised as an important way to reduce practice variation and improve the quality of care. Quality has several dimensions and this is demonstrated by the Royal College of Physicians' definition, whereby quality incorporates patient experience, effectiveness, efficiency, safety and equity. ${ }^{9}$ It frequently appears to be the case that safer and reliable systems of care delivery are also more cost-effective and efficient. ${ }^{10,11}$

\section{Identifying practice variation}

The core of any measurement initiative is a quality indicator set that combines assessments of structure, processes and outcomes of care. The choice of measures to monitor the success of quality improvement is complex. Process measures are easier to influence, while outcome measures are perceived to be more meaningful in a clinical sense - however, they can be more susceptible to case-mix variation, variance in clinical coding and care processes outside the control of the QI team. ${ }^{12,13}$

Variation in outcomes can only be readily identified by absorbing the information from several providers or facilities and comparing the results. Comparisons therefore usually take place on a regional or national level as we can see from the previously mentioned Atlas of Variation in Healthcare for People with Diabetes ${ }^{1}$ and the National Diabetes Audits. ${ }^{14}$ The audit data are publically available at CCG level and at individual practices. Examples of other measurement initiatives include the QOF, which is publically available at practice level (if you can find it), patient reported outcomes measures (PROMS) and the UK Renal Registry. ${ }^{15-17}$

\section{Interpreting and reporting variation}

Valid comparison between diabetes centres requires the collection of reliable data on factors that can affect outcomes, along with reliable clinical coding on diagnoses. 'Diabetes' is frequently missed out in HES data, as a co-morbidity when complications such as myocardial infarctions, cardiac failure or peripheral vascular disease occur - this means that relevant, important data are lost and extra income for the hospital is not claimed for.

Continuous data collection is far more preferable to episodic or annual data snapshots and is better than the traditional audit cycle. Relevant information for diabetes teams can form part of 
Box 3 Examples of clinically meaningful data

- Rates of admission for patients with diabetic emergencies

- Readmission rates for patients with problematic glycaemic control

- Length of stay for patients with diabetes

- Number of 'good diabetes days' for diabetic in-patients

- Patients with diabetes and concurrent acute coronary syndromes and stroke properly treated.

- Patient treatment satisfaction scores

- Patients attending for surgical pre-admission that have sub-optimal control diabetes identified and managed appropriately in the pre-operative phase.

- Females with diabetes and of child bearing age that have had the issue of pre-conception counselling addressed.

a continually updated clinical dashboard (see Box 3).

Awareness of this information helps focus attention on QI and performance management. Collection and graphical display of data each month enables teams to keep on track through more regular feedback of resource costs and team member performance.

It will be easier to see whether any changes in practice are directly related to a change in outcome and the use of longitudinal run-charts can allow clinical teams to assess whether changes in outcomes represent a statistically significant departure from the previous baseline or just part of normal fluctuation in outcomes.

In order for such interventions to work as reliable reporting tools, it is necessary to establish an adequate baseline score before assessing the effects of any QI intervention. There must be a reasonable interval between baseline data collection and beginning an intervention.

\section{Reducing practice variation}

Once we can establish how we are doing then we can begin to make changes. 'Trying harder' or calling for 'more training' is not guaranteed to work in the field of QI. It is changes to systems of care that require conscious adjustment. Clinicians will usually choose to concentrate on improving outcomes in which existing audits have shown performance to be poor, but may not know how to achieve improvement.

A 'change package' is a set of clinical actions or set of practices predicted to improve the outcome in question. Both of the following are good examples of quality improvement interventions.

\section{Co-creating Health}

The Health Foundation invested over $f 5$ million in a large-scale demonstration programme called Co-creating Health. This programme aimed to embed self-management support within mainstream health services across the UK and equip individuals and clinicians to work in partnership to achieve better outcomes. The Co-creating Health programme focused on developing the skills and attitudes of people with long term conditions and their clinicians, while also ensuring systems and services were designed to support and facilitate self-management. ${ }^{18}$
With regards to the impact of such a programme, in one of the pilot sites - Haringey and Islington - people living with diabetes had improved clinical outcomes (glucose control, lipids and renal function) over one year after participating in Co-creating Health.

\section{The considerative ward round checklist}

The Caldwell checklist is a list of important medical and holistic care issues that need to be addressed on a consultant medical ward round, from catheter care, to DVT prophylaxis and clarity over the working diagnosis. It has been matched to components of hospital care laid out by the GMC, NICE and the National Patient Safety Agency. This checklist encouraged documented evidence of high quality and safe medical care, and anecdotally improved team working, communication with patients, and patients' satisfaction. ${ }^{19}$ Research on the use of the checklist demonstrated an urgent need to change ward cultures to improve the professional conversations between doctors, nurses and patients. By increasing nurse presence as a result of this research, patient care and safety has improved at ward level, increasing satisfaction for everyone involved.

Relying on every individual diabetes centre to develop its own QI strategy wastes the opportunity to learn from others about the development of best practice. Arguably this is one of the roles that emergent diabetes networks need to be involved in. A good example of such a collaborative approach is the London Diabetes Foot Care Network which has been working to develop integrated care pathways for patients with 'high risk' feet, and has a steering group which includes representatives from regional clusters. ${ }^{20}$ Another is the Safer Patients Initiative funded by the Health Foundation, which was set up to test practical ways of improving hospital safety through an organisation-wide approach. ${ }^{21}$ Such collaboration also helps to identify 'outliers' and targeted intervention to aid performance management and QIP programmes.

Change packages appear to be far more manageable (or readily digested) when broken down into smaller chunks, with different teams working on different parts of the care pathway, for example the 'wicked' problem of reducing insulin-related prescribing and administration errors is best dealt with through a multi-systems approach. ${ }^{22}$ This helps to map the process of QI and assess the value that each component adds to the whole.

\section{Designing QI implementation}

There is no clear recipe for successful change as all organisations are different, but it does appear that there are some general lessons from the QI literature to be taken on board (Box 4).

These factors are almost identical to the findings from the review of diabetes oriented QI meta-analysis mentioned earlier in this paper (Box 1). There is evidence that multi-system approaches to QI are more likely to generate improvement than a single intervention. ${ }^{4,23}$ The concept of a 'care bundle' (such as the 'Sepsis Six'), all of which should be applied in a given clinical situation, is an extension of the use of evidence-based treatment protocols. Adherence to the care bundle is either 'all or none' rather than looking at each individual factor on its own. ${ }^{24}$ 


\section{Box 4 Actions to implement Q}

- Educational activities

- Audit and feedback

- Treatment protocols and algorithms

- Reminders and prompts (automated or otherwise)

- Structural changes

- Changes or revisions in staff roles and responsibilities

- Financial strategies and incentives

- Patient oriented strategies

\section{Evaluating QI}

It is more difficult to evaluate QI interventions than conventional medical treatments and there is an argument that different standards of proof should be applied. One cannot randomise and apply an intervention to multiple departments or organisations with ease as all will be unique and have their own specific quirks, barriers and problems.

There are some good examples of randomised controlled trials in the literature ${ }^{25,26}$ but other methodologies for assessment; before and after studies, performance management data, timeseries analyses or stepped wedge design (which involves sequential roll-out of an intervention to participants over a number of time periods) are also useful in providing evidence. ${ }^{27}$ There is also the issue of potentially confounding variables which can be more difficult to predict. QI interventions may be associated with a financial cost and have unintended consequences. Rigorous evaluation should always occur and ideally be ongoing.

\section{Conclusions}

Modern diabetes care is complex and involves multiple interdependencies. It is delivered by different teams in different settings to different standards (both literally and figuratively).

Conscious redesign of the way in which teams interact is very important. A recipe for successful patient management based on key QI principles means that we can provide reliable, efficient, protocol-driven care. There are still those who believe that each individual doctor should be able to decide the best treatment for 'their patient' in each and every clinical situation, based on their own critical analysis of the literature - but this is becoming an outdated view. Our efforts should perhaps be spent on learning when a given situation justifies departing from 'the way we do it round here' and looking at the entire system of care.

\section{Conflict of interest None \\ Funding sources None}

Acknowledgements Many thanks to Professor Stuart Whittaker at COHSASA and Derek Lington.

\section{References}

1. NHS Atlas of Variation in Healthcare for People with Diabetes. http://www.rightcare.nhs.uk/index.php/atlas/diabetes/ (Accessed March 2013).

2. Mathews SC, Pronovost PJ. Physician autonomy and informed decision making: finding the balance for patient safety and quality. JAMA 2008;300:2913-5. http://dx.doi.org/10.1001/jama.2008.846

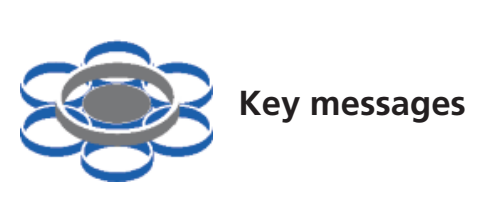

- There are clear variations in the quality and processes of care delivered on a local and regional level

- Quality improvement initiatives are required to ensure that high standards are achieved and maintained in order to reduce variation in outcomes

- Commitment is required by diabetes stakeholders to identify and overcome the barriers to QI implementation

3. Tomson, CRV. Van der Veer SN. Learning from practice variation to improve the quality of care. Clin Med 2012;13:19-23.

http://dx.doi.org/10.7861/clinmedicine.13-1-19

4. Tricco AC, Ivers NM, Grimshaw JM, et al. Effectiveness of quality improvement strategies on the management of diabetes: a systematic review and meta-analysis. Lancet 2012;379:2252-61.

http://dx.doi.org/10.1016/S0140-6736(12)60480-2

5. O'Connor P. Quality improvement collaboratives in the age of health informatics-new wine in new wineskins. BMJ Qual Saf 2012;21:891-3. http://dx.doi.org/10.1136/bmjgs-2012-001265

6. NICE Quality Standard for Diabetes in Adults QS6 March 2011. http://guidance.nice.org.uk/QS6 (accessed March 2013).

7. Grant P. The New NICE Quality Standards for Diabetes 2011: a critical analysis. Pract Diab Int 2011;28:208. http://dx.doi.org/10.1002/pdi.1594

8. Hazlehurst JM, Armstrong MJ, Sherlock M, et al. A comparative quality assessment of evidence-based clinical guidelines in endocrinology. Clin Endocrinol 2013;78:183-90.

http://dx.doi.org/10.1111/j.1365-2265.2012.04441.x

9. Atkinson $S$, Ingham J, Went $S$, et al. Defining quality and quality improvement. Clin Med 2010;10:537-9.

http://dx.doi.org/10.7861/clinmedicine.10-6-537

10. Echouffo-Tcheugui JB, Dagogo-Jack S. Preventing diabetes mellitus in developing countries. Nat Rev Endocrinol 2012;8:557-62. http://dx.doi.org/10.1038/nrendo.2012.46

11. Beard EL Jr, Sharkey K. Innovation amidst radical cost containment in health care. Nurs Adm Q 2013;37:116-21. http://dx.doi.org/10.1097/NAQ.0b013e3182869e67

12. Bombardier C, Mian S. Quality indicators in rheumatoid arthritis care: using measurement to promote quality improvement. Ann Rheum Dis 2013;72:Suppl 2:ii128-ii131. http://dx.doi.org/10.1136/annrheumdis-2012-202259

13. Hysong SJ, Khan MM, Petersen LA. Passive monitoring versus active assessment of clinical performance: impact on measured quality of care Med Care 2011;49:883-90. http://dx.doi.org/10.1097/MLR. Ob013e318222a36c

14. National Diabetes Audit. Health \& Social Care Information Centre, http://www.hscic.gov.uk/nda. (Accessed May 2013).

15. Quality and Outcomes Framework. Online GP practice results database, http://www.qof.ic.nhs.uk/ (Accessed June 2013).

16. Patient Reported Outcome Measures. Health \& Social Care Information Centre, http://www.hscic.gov.uk/proms (Accessed June 2013).

17. UK Renal Registry. The Renal Association, http://www.renalreg.com/ Accessed June 2013.

18. Co-creating health. The Health Foundation, http://www.health.org.uk/ areas-ofwork/programmes/co-creating-health/impact/ (Accessed June 2013).

19. Herring R, Caldwell G, Jackson S. Implementation of a considerative checklist to improve productivity and team working on medical wards rounds. Clin Governance 2011;16:129-36. http://dx.doi.org/10.1108/14777271111124482 
20. London Diabetes Footcare Network. NHS Diabetes, http://www.diabetes.nhs.uk/networks/footcare_network/pan_london_diabetic_footcare_network_meeting_sept_2012/(Accessed June 2013).

21. Safer Patient Initiative. The Health Foundation, http://www. health.org.uk/areas-ofwork/programmes/safer-patients-initiative/ (Accessed June 2013)

22. Grant P, Mustafa O, Malik R. Setting up an insulin safety group - A practical approach. Practical Diabetes 2012;29:160-2. http://dx.doi.org/10.1002/pdi.1682

23. Beitsch LM, Carretta H, McKeever J, et al. The quantitative story behind the quality improvement storyboards: a synthesis of quality improvement projects conducted by the multi-state learning collaborative. J Public Health Manag Pract 2013;19:330-40.

http://dx.doi.org/10.1097/PHH.0b013e3182629054
24. Surviving Sepsis Campaign Care Bundles, http://www survivingsepsis.org/Bundles/Pages/default.aspx (Accessed June 2013).

25. Mate KS, Ngidi WH, Reddy J, et al. A case report of evaluating a largescale health systems improvement project in an uncontrolled setting: a quality improvement initiative in KwaZulu-Natal, South Africa. BMJ Qual Saf 2013:22(11):891-8

26. Williamson $M$, Cardona-Morrell M, Elliott JD et al. Prescribing Data in General Practice Demonstration (PDGPD) project-a cluster randomised controlled trial of a quality improvement intervention to achieve better prescribing for chronic heart failure and hypertension. BMC Health Serv Res 2012;12:273. http://dx.doi.org/10.1186/1472-6963-12-273.

27. Brown CA, Lilford RJ. The stepped wedge trial design: a systematic review. BMC Med Res Methodol 2006,6:54.

http://dx.doi.org/10.1186/1471-2288-6-54 